Research Article

\title{
Single Snapshot DOA Estimation by Minimizing the Fraction Function in Sparse Recovery
}

\author{
Changlong Wang, Jibin Che $\mathbb{C}^{\mathrm{D}}$, Feng Zhou, Jinyong Hou, and Chen Li \\ Key Laboratory of Electronic Information Countermeasure and Simulation Technology, Ministry of Education Xidian University, \\ $X i$ 'an, China \\ Correspondence should be addressed to Jibin Che; chejiben@126.com
}

Received 6 July 2020; Accepted 27 July 2020; Published 24 August 2020

Guest Editor: Jianfeng Li

Copyright (c) 2020 Changlong Wang et al. This is an open access article distributed under the Creative Commons Attribution License, which permits unrestricted use, distribution, and reproduction in any medium, provided the original work is properly cited.

\begin{abstract}
Sparse recovery is one of the most important methods for single snapshot DOA estimation. Due to fact that the original $l_{0}$-minimization problem is a NP-hard problem, we design a new alternative fraction function to solve DOA estimation problem. First, we discuss the theoretical guarantee about the new alternative model for solving DOA estimation problem. The equivalence between the alternative model and the original model is proved. Second, we present the optimal property about this new model and a fixed point algorithm with convergence conclusion are given. Finally, some simulation experiments are provided to demonstrate the effectiveness of the new algorithm compared with the classic sparse recovery method.
\end{abstract}

\section{Introduction}

The problem of estimating the direction of arrival (DOA) of signals impinging on an array of sensors is widely applied in radar, sonar, and wireless communication systems [1-9]. For fast-moving sources and multipath propagation problems, snapshots are limited, so high resolution adaptive DOA estimation approaches such as MVDR [10], MUSIC [11], and covariance matching methods $[12,13]$ fail due to inaccurate estimation of the spatial covariance matrix.

As one of the most important methods designed for single snapshot DOA estimation, sparse recovery has its own advantage for single snapshot case [14-17]. By dividing the angle range into grid points, the number of source is much less than that of grid points. By matching the grid points, these methods usually consider to solve the following $l_{0}$-minimization:

$$
\begin{array}{ll}
\min & \|x\|_{0} \\
\text { s.t. } & A x=y,
\end{array}
$$

where $\|\cdot\|_{0}$ stands for the number of nonzero elements. In recent years, many sparse algorithms such as OMP and $l_{1}$-minimization [18-22] have been applied to solve this problem. Although a lot of work has given the rationality of sparse recovery algorithms [23-25], these conditions not only require the measurement matrix to meet the RIP condition, but also the corresponding RIC constant to meet certain conditions. However, verifying RIP conditions for a given matrix is itself an NP-hard problem, and the current RIP estimation conclusion is only valid for random matrices. However, DOA measurement matrix does not have such a random structure, so it is difficult to directly verify its RIP conditions. Therefore, there is no sufficient guarantee of model theory. In order to overcome these difficulties, we use the following fraction function:

$$
g_{p}(x)=\frac{|x|}{\sqrt{x^{2}+p}}
$$

to replace the original 0 -norm. In Figure 1, it is easy to get that the new alternative function tends to 0 -norm when $p \longrightarrow 0$. Therefore, it is reasonable to believe this function is a good choice. 


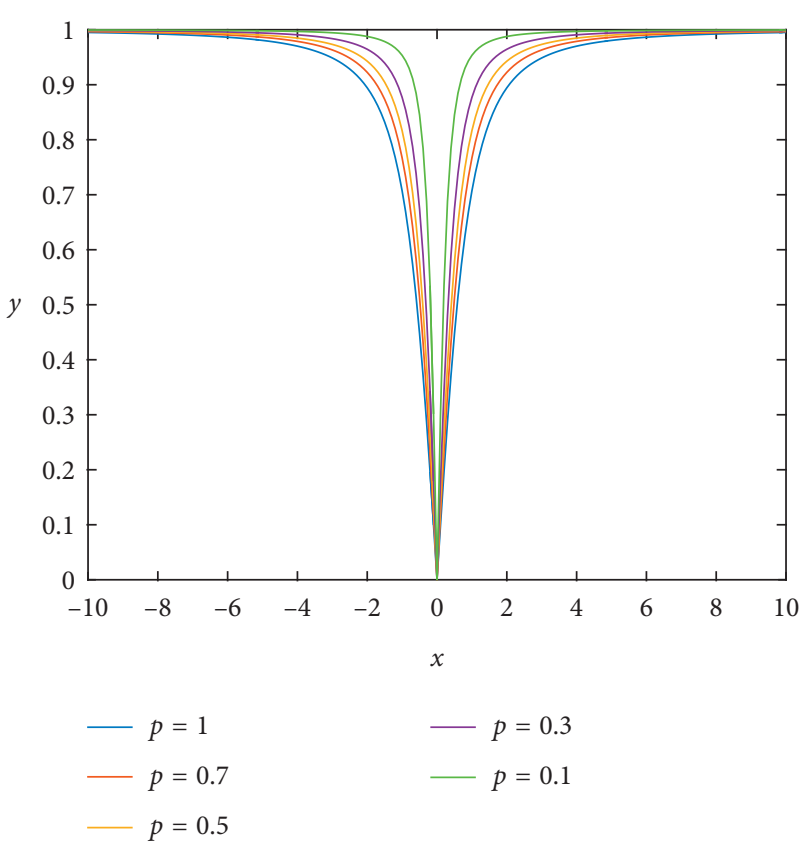

FIgURE 1: The graph of $g_{p}(x)$ with different $p$.

1.1. The Existed Main Problems of Classic Sparse DOA Estimation Methods. As the main idea of these methods, the real DOA is recovered by matching the real solution with the grid points. In order to deal with off-grid cases, we have to reduce the spacing between these points and increase the number of grid points. If we do not care about the hardware, the main problem caused by a big number of grid points is the measurement matrix $A$ with high coherence $\mu(A)$ which leads to invalidation of the existed sparse methods, such as $l_{1}$-minimization and OMP.

For a given matrix $A$, the coherence $\mu(A)$ is defined as

$$
\mu(A)=\max _{i \neq j} \frac{A_{i}^{T} A_{j}}{\left\|A_{i}\right\|_{2}\left\|_{j}\right\|_{2}} .
$$

In [24], OMP can recover the real sparse solution as long as the number of sources $k$ satisfies the following inequalities:

$$
k<\frac{1}{2}\left(1+\frac{1}{\mu(A)}\right) \text {. }
$$

Meanwhile, it is obvious that the coherence will increase as long as the number of grid points increases. In Figure 2, the coherence of measurement matrices changes as the number of grid points increases, and it is obvious that OMP only can guarantee one source when the number of grid points is more than ten.

Besides, increasing the number of grid points also lead to the RIP condition deterioration of the measurement matrix. A matrix $A$ is said to satisfy RIP of order $2 k$ if and only if there exists a constant $\delta_{2 k} \in(0,1)$ such that

$$
\left(1-\delta_{2 k}\right)\|x\|_{2}^{2} \leq\|A x\|_{2}^{2} \leq\left(1+\delta_{2 k}\right)\|x\|_{2}^{2}
$$

for any $2 k$ sparse vector $x$. It is obvious that $\delta_{2 k}$ increases as the number of grid points increases until RIP is no longer

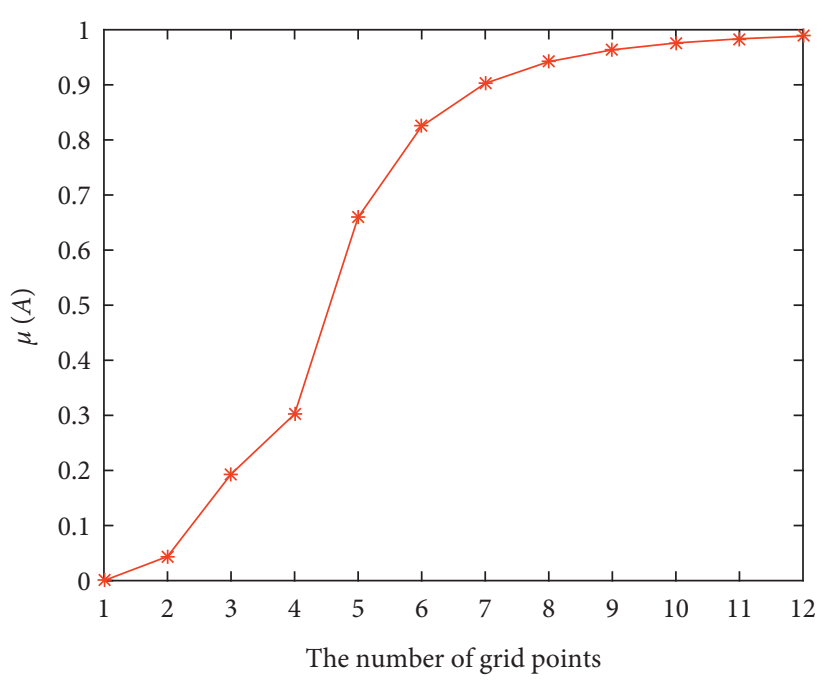

FIgURE 2: The relationship between $\mu(A)$ and the number of grid points.

satisfied. For the alternative method, [25] has proved that $k$ sources can be recovered as long as $\delta_{2 k} \leq(\sqrt{2} / 2)$, and this condition is theoretical optimal.

Therefore, both greedy algorithms and $l_{1}$-minimization are difficult to deal with situation when the number of grid points increases.

1.2. The Main Contribution of This Paper. To summarize, the main contribution of this paper can be expressed as follows:

(1) In order to design a reasonable sparse recovery model for solving DOA estimation, we use the alternative function $g_{p}(\cdot)$ to replace 0 -norm and give some theoretical analysis about the new alternative function

(2) With theoretical guarantee about the new alternative model, we design a fixed iterative algorithm and the convergence conclusion is also given

This paper is organized as follows. In Section 2, we review the model processing of DOA estimation problem and give the upper number of sources that the sparse method can recover. We give a new alternative function $g_{p}(\cdot)$ for $l_{0}$-minimization and prove the equivalence between the new models and the original sparse model both in noiseless and noise cases. By analysing the optimal property about this new model in Section 3, an algorithm designed for minimizing the fraction function $g_{p}(\cdot)$ and its convergence are presented. Some simulation experiments are given. Compared to some classic methods, the proposed method has a better result than others.

1.3. Symbols. Through this paper, we use $\theta^{*} \in \mathbb{R}^{K}$,

$$
\theta^{*}=\left\{\alpha_{1}, \alpha_{2}, \ldots, \alpha_{K}\right\}
$$

which stands for the real DOA solution, and use $\Theta \in \mathbb{R}^{n}$, 


$$
\Theta=\left\{\theta_{1}, \theta_{2}, \ldots, \theta_{n}\right\},
$$

which stands for the grid point sets. For convenience, for $x \in \mathbb{R}^{n}$, its support is defined by $\operatorname{suppt}(x)=\left\{i: x_{i} \neq 0\right\}$ and the cardinality of set $\Omega$ is denoted by $|\Omega|$. Let $\operatorname{Ker}(A)=$ $\left\{x \in \mathbb{R}^{n}: A x=0\right\}$ be the null space of the matrix $A$. We define subscript notation $x_{\Omega}$ to be such a vector that is equal to $x$ on the index set $\Omega$ and zero everywhere else and use the subscript notation $A_{\Omega}$ to denote a submatrix whose columns are those of the columns of $A$ that are in the set index $\Omega$. Let $\Omega^{c}$ be the complement of $\Omega$. For any positive integer $n$, we denote $[n]=\{1,2,3, \ldots, n\}$.

\section{Sparse DOA Estimation Model and Some Theory Analysis}

2.1. Data Model. Assume that $K$ far-field stationary and narrowband signals impinge on an $M$-element uniform linear array with DOAs of $\Theta^{*}=\left[\theta_{1}, \theta_{2}, \ldots, \theta_{K}\right]$. For a given $\theta \in \mathbb{R}$, define a vector $A(\theta) \in \mathbb{R}^{M}$,

$$
A(\theta)_{m}=\exp \left(-j 2 \pi(m-1)\left(\frac{d \cdot \sin \theta}{\lambda}\right)\right),
$$

where $m \in[M]$ and where $d$ is the distance between adjacent sensors and $\lambda$ is the wavelength of the incident signals. Then, the array outputs of $N$ snapshots can be expressed as

$$
y(t)=A\left(\Theta^{*}\right) s(t)+n(t), \quad t=1,2, \ldots, N,
$$

where $s(t)=\left[s_{1}(t), s_{2}(t), \ldots, s_{K}(t)\right]^{T}$ stands for far-field signals and $n(t)$ stands for the noise vector. $A\left(\Theta^{*}\right)$ is an $M \times K$ array manifold matrix, whose elements

$$
A\left(\Theta^{*}\right)=\left[A\left(\theta_{1}\right), A\left(\theta_{2}\right), \ldots, A\left(\theta_{K}\right)\right] .
$$

We consider to recover $\theta^{*}$ from a grid points set $\Omega=\left\{\alpha_{1}, \alpha_{2}, \ldots, \alpha_{n}\right\} \in \mathbb{R}^{n}$. If $\Theta^{*} \subseteq \Theta$, then there exist two mappings $\pi_{\Theta^{*}, \Omega}:[K] \longrightarrow[n]$ and $\mu_{\Theta^{*}, \Omega}: \Theta^{*} \longrightarrow \Omega$ such that

$$
u_{\Theta^{*}, \Omega}\left(\theta_{i}\right)=\alpha_{\pi_{\Theta^{*}, \Omega}(i)} .
$$

It is obvious that

$$
A(\Theta) x^{*}(t)=y(t)
$$

where

$$
x^{*}(t)_{i}= \begin{cases}s_{\pi_{\Theta^{*}, \Omega}(i)}(t), & i \in \pi_{\Theta^{*}, \Omega}([K]), \\ 0, & \text { else. }\end{cases}
$$

Once $n \gg M$ and $N=1$, we can recover $\Theta^{*}$ via the following $l_{0}$-minimization:

$$
\begin{array}{ll}
\min _{x \in \mathbb{R}^{n}} & \|x\|_{0} \\
\text { s.t. } & A(\Omega) x=y(t) .
\end{array}
$$

Then, we can recover $\Theta^{*}$ by $\operatorname{supp}(x)=\left\{i \in[n] \mid\left\|x_{i}\right\|_{2}>0\right\}$.
2.2. Sparse DOA Estimation via Minimizing Fraction Function. As one of the most important methods designed for single snapshot DOA estimation, the following theorem shows the upper bound on the number of sources by the sparse method.

Theorem 1. For the measurement matrix $A(\Omega) \in \mathbb{C}^{M \times n}$ defined in (10), if $\theta^{*} \subseteq \Theta, K<((M+1) / 2)$ and there is no noise during the measurement; then, the real solution $\Theta^{*}$ of $D O A$ estimation can be recovered by $l_{0}$-minimization (14), i.e.,

$$
\Theta^{*}=\left\{\alpha_{i} \mid i \in \operatorname{supp}(x)\right\},
$$

where $x$ is the solution of model (14).

Proof. Without loss of generality, we consider the following vector $x^{*} \in \mathbb{R}^{n}$ :

$$
x_{i}^{*}= \begin{cases}s_{\pi_{\Theta^{*}, \Omega}(i)}, & i \in \pi_{\Theta^{*}, \Omega}([K]), \\ 0, & \text { else. }\end{cases}
$$

It is obvious that $A(\Omega) x^{*}=y$ and $\left\|x^{*}\right\|_{0} \leq K$. Therefore, it is enough to prove that $x^{*}$ is the sparsest solution of $A(\Omega) x^{*}=y$.

If there exists another solution $z^{*} \in \mathbb{R}^{n}$ such that

$$
\begin{aligned}
A(\Omega) z^{*} & =y, \\
\left\|z^{*}\right\|_{0}<\left\|x^{*}\right\|_{0} & \leq K .
\end{aligned}
$$

Therefore, we can get that $z^{*}-x^{*} \in \operatorname{Ker}(A(\Omega))$ and

$$
\left\|z^{*}-x^{*}\right\|_{0} \leq\left\|z^{*}\right\|_{0}+\left\|x^{*}\right\|_{0} \leq 2 K \leq M,
$$

since $K<M+(1 / 2)$.

However, for the given Vandermonde matrix $A(\Omega)$, it is obvious that any of its submatrix of order $M$ is a full-rank matrix; i.e., for $\forall S \in[n]$, we have that

$$
\left|A(\Omega)_{S}\right|=\prod_{\substack{u, v \in S \\ u<v}}\left(\exp \left(\frac{-j 2 \pi d \cdot \sin \theta_{u}}{\lambda}\right)-\exp \left(\frac{-j 2 \pi d \cdot \sin \theta_{v}}{\lambda}\right)\right) \neq 0,
$$

since $\theta_{u} \neq \theta_{v}$, which is contradict conclusion (18).

By Theorem 1, the performance of the sparse recovery method is clearly demonstrated. In practices, we usually consider the following model because of noise:

$$
\begin{array}{ll}
\min _{x \in \mathbb{R}^{n}} & \|x\|_{0} \\
\text { s.t. } & \|A(\Omega) x-y\|_{f} \leq \varepsilon,
\end{array}
$$

where $\|\cdot\|_{f}$ stand for a certain norm. Similar to discussion above, in this paper, we use the following $l_{g_{p}}^{f}(\varepsilon)$-minimization model instead of (20):

$$
\begin{array}{ll}
\min _{x \in \mathbb{R}^{n}} & \|x\|_{g_{p}} \\
\text { s.t. } & \|A(\Omega) x-y\|_{f} \leq \varepsilon .
\end{array}
$$


Before, we prove the equivalence between (20) and (21), some lemmas are needed. The following lemma is easy to get by the definition of $\|\cdot\|_{0}$, and we leave the proof to the readers.

Lemma 1. If $x^{*}$ is the solution of $l_{0}$-minimization (22), then the column vectors belong to $\operatorname{supp}\left(x^{*}\right)$ are linearly independent.

Lemma 2. If $x^{*}$ is the solution of $l_{g_{p}}^{f}(\varepsilon)$-minimization (23), then the column vectors belong to $\operatorname{supp}\left(x^{*}\right)$ are linearly independent.

Proof. If the submatrix $A_{\text {supp }\left(x^{*}\right)}$ is not full rank, then there exists a vector $h \in \operatorname{Ker}(A) \operatorname{such}$ that $\operatorname{supp}(h) \subseteq \operatorname{supp}\left(x^{*}\right)$. For such $x^{*}$ and $h$, let

$$
a=\max _{h_{i} \neq 0} \frac{\left|x_{i}^{*}\right|}{\left|h_{i}\right|} .
$$

Therefore, it is easy to get that

$$
\operatorname{sgn}\left(x_{i}^{*}+\alpha h_{i}\right)=\operatorname{sgn}\left(x_{i}^{*}-\alpha h_{i}\right)=\operatorname{sgn}\left(x_{i}^{*}\right),
$$

for $\alpha \in[0, a]$ with $x_{i}^{*} \neq 0$.

Since $g_{p}(x)$ is a concave function when $x \geq 0$, it is easy to get that

$$
\begin{aligned}
g_{p}\left(x_{i}^{*}\right)= & g_{p}\left(\frac{1}{2} x_{i}^{*}+\alpha h_{i}+\frac{1}{2} x_{i}^{*}-\alpha h_{i}\right) \geq \frac{1}{2} g_{p}\left(x_{i}^{*}+\alpha h_{i}\right) \\
& +\frac{1}{2} g_{p}\left(x_{i}^{*}-\alpha h_{i}\right) .
\end{aligned}
$$

Therefore, we can get that

$$
g_{p}\left(x_{i}^{*}\right) \geq \frac{1}{2} g_{p}\left(x_{i}^{*}+\alpha h_{i}\right)+\frac{1}{2} g_{p}\left(x_{i}^{*}-\alpha h_{i}\right),
$$

and it is easy to get that

$$
\left\|x^{*}\right\|_{g_{p}} \geq \frac{1}{2}\left\|x^{*}+\alpha h\right\|_{g_{p}}+\frac{1}{2}\left\|x^{*}-\alpha h\right\|_{g_{p}}
$$

which contradicts the assumptions.

In order to extend of application of $g_{p}(\cdot)$ in sparse recovery, we consider the following models, $F_{0}(r)$-minimization and $F_{g_{p}}(r)$-minimization. Furthermore, $(20)$ and (21) can be treated as special cases of these two models:

$$
\begin{array}{ll}
\text { min } & \|x\|_{0} \\
\text { s.t. } & \left\{\begin{array}{l}
A x \leq b, \\
\|x\|_{\infty} \leq r,
\end{array}\right. \\
\text { min } & \|x\|_{g_{p}} \\
\text { s.t. } & \left\{\begin{array}{l}
A x \leq b, \\
\|x\|_{\infty} \leq r .
\end{array}\right.
\end{array}
$$

Next, the following theorem shows the equivalence between $F_{0}(r)$-minimization and $F_{g_{p}}(r)$-minimization.
Theorem 2. For any $A, b$, and $r$, there exists a constant $p^{*}(A, b, r)$ such that the solution of $F_{g_{p}}(r)$-minimization (28) also solves $F_{0}(r)$-minimization whenever $0<p<p^{*}(A, b, r)$.

Proof. It is easy to get the constraint region in model (27) and model (28) are polygons which is a convex combination of its limited extreme points.

Define a set $V=\left\{v \in \mathbb{R}^{n} \mid v_{i} \in\{-1,0,1\}\right\}$, and $\|\cdot\|_{g_{p}}$ is a concave function for a given quadrant $\{x \mid \operatorname{sgn}(x)=\nu$, $v \in V\}$ so the solution of the following problem must be contained in the extreme points of the convex polygon $\left\{x \in \mathbb{R}^{n} \mid \operatorname{sgn}(x)=v, A x \leq y,\|x\|_{\infty} \leq r\right\}:$

$$
\begin{array}{ll}
\min _{\operatorname{sgn}(x)=v} & \|x\|_{g_{p}} \\
\text { s.t. } & \left\{\begin{array}{l}
A x \leq y, \\
\|x\|_{\infty} \leq r .
\end{array}\right.
\end{array}
$$

Since the number of quadrants in $\mathbb{R}^{n}$ is limited, so there exists a limited point set $\widehat{V}$ such that model (28) is equal to

$$
\begin{array}{ll}
\min _{x \in \widehat{V}} & \|x\|_{g_{p}} \\
\text { s.t. } & \left\{\begin{array}{l}
A x \leq y, \\
\|x\|_{\infty} \leq r .
\end{array}\right.
\end{array}
$$

For such limited points set $\widehat{V}$, define its subset $V^{*}$ as

$$
V^{*}=\left\{x \in \widehat{V} \mid\|x\|_{0} \leq\|y\|_{0}, \forall y \in \widehat{V}\right\} .
$$

Since $\|\cdot\|_{g_{p}}$ is a continuous function and the element number of $\widehat{V}$ is limited, we can define a constant $p^{*}(A, y, r)$ such that

$$
\|x\|_{g_{p}}<\|y\|_{g_{p}},
$$

for any $x \in V^{*}, y \in \widehat{V} \backslash V^{*}$, and $0<p<p^{*}(A, y, r)$. Finally, it is obvious that the elements of $V^{*}$ also solve $F_{0}(r)$-minimization (27) since $\|\cdot\|_{g_{p}}$ is a continuous function.

The proof is completed.

Corollary 1. For the noiseless cases and the measurement matrix $A(\Omega) \in \mathbb{C}^{M \times n}$ defined in (10), if $\Theta * \subseteq \Omega$ and $K<((M+1) / 2)$, then there exists a constant $p^{*}(A, y)$ such that the real solution $\Theta *$ of DOA estimation can be recovered by both model (20) and model (21) whenever $0<p<p^{*}(A, y)$.

Proof. By Lemma 2, it is obvious that both of model (20) and model (21) are equal to the themselves with a bounded constrained $\|x\|_{\infty} \leq T(A(\Omega), y)$, where

$$
T(A(\Omega), y)=\min _{x_{i} \neq 0}\left\{x_{i} \mid A(\Omega) x=y, \operatorname{rank}\left(A(\Omega)_{\operatorname{supp}(x)}\right)=\|x\|_{0}\right\} .
$$

Since the solution $x$ of $A x=y$ with $A_{\operatorname{supp}(x)}=\|x\|_{0}$ is limited, it is impossible to calculate $T(A(\Omega), y)$ for given $A(\Omega)$ and $y$. 
By Theorem 1, we can conclude the equivalence between model (27) and model (28), and the proof is completed.

Corollary 2. Let $f=1$ or $f=\infty$, then there exists a constant $p_{f}^{*}(A, b, \varepsilon)$ such that the solution of $l_{g_{p}}^{f}(\varepsilon)$-minimization (28) also solves $l_{g_{p}}^{f}(\varepsilon)$-minimization (27) whenever $0<p<p_{f}^{*}(A, b, \varepsilon)$

Proof. By the prove in Theorem 1, it is enough to prove the constraint zone in (27) and (28) are polytopes. By Lemmas 1 and 2 and (33), it is easy to find that the solutions of (27) and (28) are contained in a bounded zone.

When $f=1$ or $f=\infty$, the constraint zone $\|A x-y\|_{f}$ can be rewritten as

$$
\begin{aligned}
& \Delta(A x-y) \leq \varepsilon 1, \\
& -\varepsilon 1+y \leq A x \leq \varepsilon 1+y,
\end{aligned}
$$

where the matrix $\Delta \in \mathbb{R}^{2^{n} \times n}$ with $\Delta_{i, j} \in\{-1,1\}$ stands for the whole permutations by $-1,1$.

The proof is completed.

\section{A Sparse Recovery Algorithm Designed for DOA Estimation}

In Section 2, we show the theoretical performance of DOA sparse methods and the equivalence between the alternative function $g_{p}(\cdot)$ and the original 0-norm. In this section, we will focus on the algorithm designed for DOA estimation. As the theoretical basis for new algorithm, the following theorem shows us the local property of $l_{g_{p}}$-minimization.

Theorem 3. If $\Theta^{*} \subseteq \Omega, \quad 2 K<m+1, \quad$ and $0<p<p^{*}(A(\Omega), y)$, then $x^{*}$ is the solution of model (14):

$$
x_{i}^{*}= \begin{cases}s_{\pi_{\Theta^{*}, \Omega}(i)}, & i \in \pi_{\Theta^{*}, \Omega}([K]), \\ 0, & \text { else, }\end{cases}
$$

and $x^{*}$ satisfies the following equalities:

$$
x^{*}=\Gamma\left(x^{*}\right) A^{T}\left(A \Gamma\left(x^{*}\right) A^{T}\right)^{\dagger} y,
$$

where $\Gamma(x)$ is a diagonal matrix with $\Gamma(x)_{i, i}=\left(\left|x_{i}\right|\left(x_{i}^{2}+p\right)^{1.5} / p\right)$.

Proof. Without loss of generality, we assume that $\operatorname{supp}(x)=$ $[K]$ and consider the following problem:

$$
\begin{array}{ll}
\min _{t \in \mathbb{R}^{s}} & \|t\|_{g_{p}} \\
\text { s.t. } & B t=y,
\end{array}
$$

where $B=A(\Omega)_{[K]}$. It is obvious that $x_{[K]}$ is the solution of model (37) and there exists a constant $\eta$ small enough such that the function $\|\cdot\|_{g_{p}}$ is differentiable at the point $x=x_{[K]}$ when $\left\|t-x_{[K]}\right\|_{2} \leq \eta$. Therefore, KKT condition can be applied in such area. Define the Lagrange function $L(t, \lambda)$ as follows:

$$
L(t, \lambda)=\|t\|_{g_{p}}-\lambda^{T}(B t-y) .
$$

Therefore, $x_{[K]}$ must be the solution of the following equations:

$$
\left\{\begin{array}{l}
\frac{\partial L}{\partial x} \mid=0 \\
B t=y
\end{array}\right.
$$

To solve equation (39), we can get that

$$
\begin{aligned}
\lambda^{*} & =\left(B F(z) B^{T}\right)^{-1} b, \\
x_{[K]} & =\Gamma\left(x_{[K]}\right) B^{T}\left(B \Gamma\left(x_{[K]}\right) B^{T}\right)^{-1} y .
\end{aligned}
$$

Since $\operatorname{supp}\left(x^{*}\right)=[K]$, it is easy to get that

$$
x=\Gamma(x) A(\Omega)^{T}\left(A(\Omega) \Gamma(x) A(\Omega)^{T}\right)^{-\dagger} y .
$$

By analysis expression (36), a fixed point iterative algorithm is presented in Algorithm 1. Next, the following theorem shows the convergence conclusion of this new algorithm.

Theorem 4. The sequence $\left\{x^{k}\right\}$ produced by

$$
x^{k+1}=\Gamma\left(x^{k}\right) A^{T}\left(A \Gamma\left(x^{k}\right) A^{T}\right)^{\dagger} y,
$$

satisfies the following equality:

$$
\left\|x^{k+1}\right\|_{g_{p}} \leq\left\|x^{k}\right\|_{g_{p}}
$$

and the limit point $x^{*}$ satisfies equality (36).

Proof. Since $x^{k+1}=\Gamma\left(x^{k}\right) A^{T}\left(A \Gamma\left(x^{k}\right) A^{T}\right)^{\dagger} y$, it is obvious that

$$
\left\{\begin{array}{l}
\Gamma\left(x^{k}\right)^{\dagger} x^{k+1}=A^{T}\left(A \Gamma\left(x^{k}\right) A^{T}\right)^{\dagger} y, \\
A x^{k+1}=y .
\end{array}\right.
$$

Therefore, we can conclude that $x^{k+1}$ is the solution of the following problem:

$$
\begin{array}{ll}
\min _{x} & x^{T} \Gamma\left(x^{k}\right)^{\dagger} x \\
\text { s.t. } & A x=y,
\end{array}
$$

i.e.,

$$
\sum_{x_{i}^{k} \neq 0} \frac{g_{p}\left(x_{i}^{k}\right)}{\left|x_{i}^{k}\right|}\left(x_{i}^{k+1}\right)^{2} \leq \sum_{x_{i}^{k} \neq 0} g_{p}\left(x_{i}^{k}\right)\left|x_{i}^{k}\right| .
$$

By the expression of $g_{p}(\cdot)$, it is easy to get that

$$
g_{p}\left(x_{i}^{k+1}\right)-\frac{g_{p}^{\prime}\left(x_{i}^{k}\right)\left(x_{i}^{k+1}\right)^{2}}{2\left|x_{i}^{k}\right|} \leq g_{p}\left(x_{i}^{k}\right)-\frac{\left|x_{i}^{k}\right| g_{p}^{\prime}\left(x_{i}^{k}\right)}{2} \text {. }
$$

By (46) and (47), we can conclude that

$$
\left\|x^{k+1}\right\|_{g_{p}} \leq\left\|x^{k}\right\|_{g_{p}} .
$$




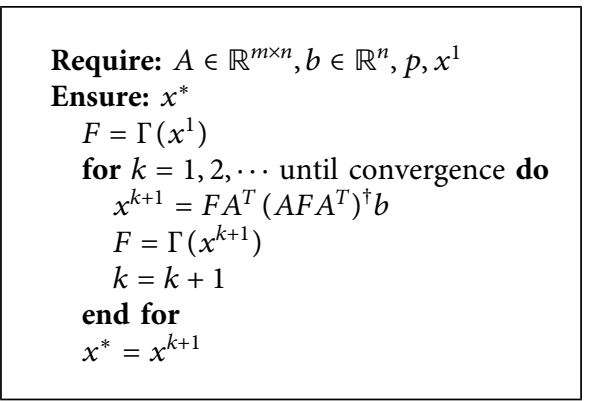

Algorithm 1: An sparse recovery algorithms for DOA estimation.

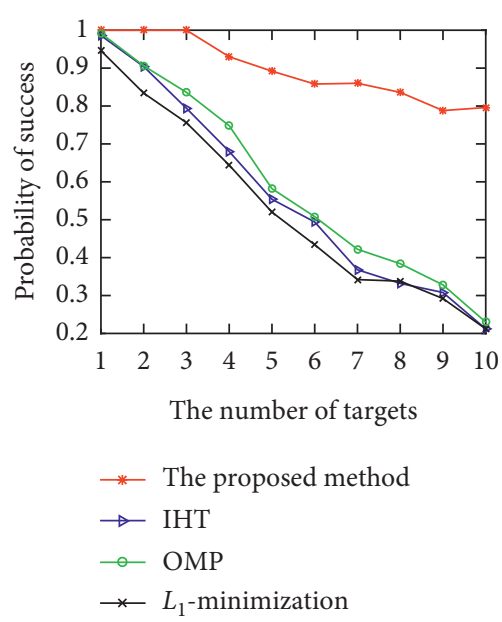

(a)

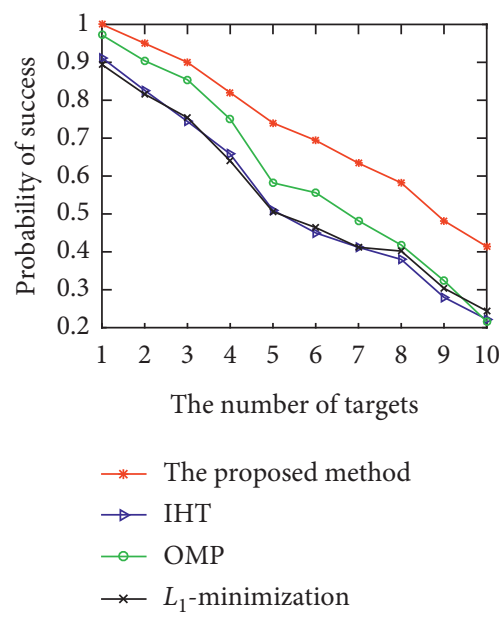

(d)

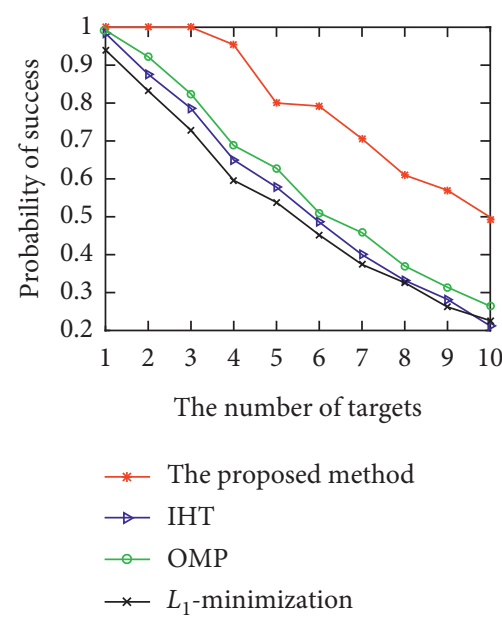

(b)

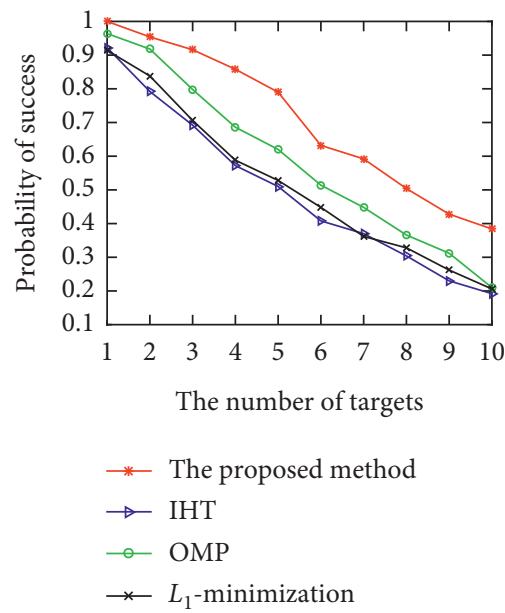

(e)

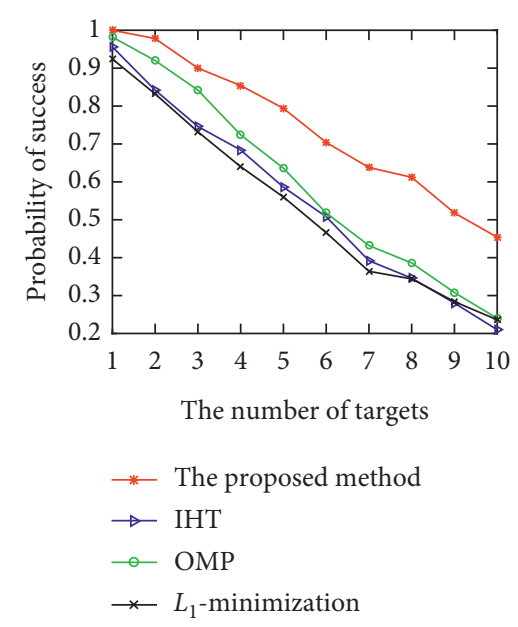

(c)

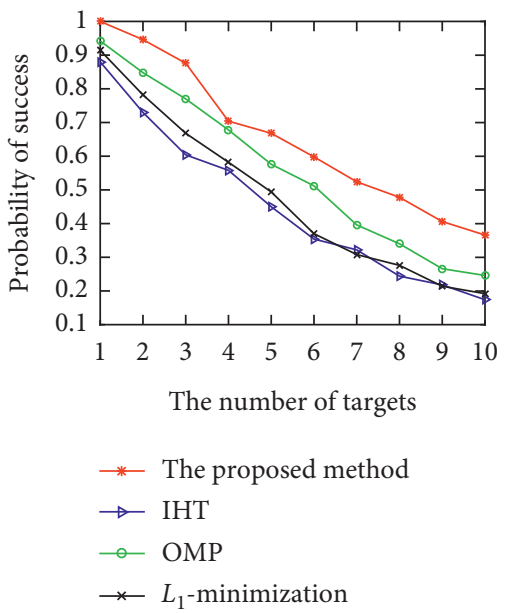

(f)

FIgURE 3: The result of the proposed method and classic method: (a) $80 \mathrm{~dB}$, (b) $20 \mathrm{~dB}$, (c) $15 \mathrm{~dB}$, (d) $10 \mathrm{~dB}$, (e) $5 \mathrm{~dB}$, and (f) $0 \mathrm{~dB}$.

Since $\|\cdot\|_{g_{p}} \geq 0$ and $\|x\|_{g_{p}}=0$ if and only if $x=0$, the sequence $\left\|x^{k+1}\right\|_{g_{p}}$ is convergent and the limit point $x^{*}$ is the solution of

$$
\begin{array}{ll}
\min _{x} & x^{T} \Gamma\left(x^{*}\right)^{\dagger} x \\
\text { s.t. } & A x=y .
\end{array}
$$

By the Lagrange function of (49), we can get the conclusion of this theorem.

Next, we will give some experiment results to show the effective of the proposed method. In Figure 3, we consider the case when $\Theta^{*} \subseteq \Omega$. In this experiments, we take $M=40$ and $n=180$, and the range of angle is $\left[-90^{\circ}, 90^{\circ}\right]$. Under different SNR $80 \mathrm{~dB}, 20 \mathrm{~dB}, 15 \mathrm{~dB}, 10 \mathrm{~dB}, 5 \mathrm{~dB}$, and $0 \mathrm{~dB}$, it is 


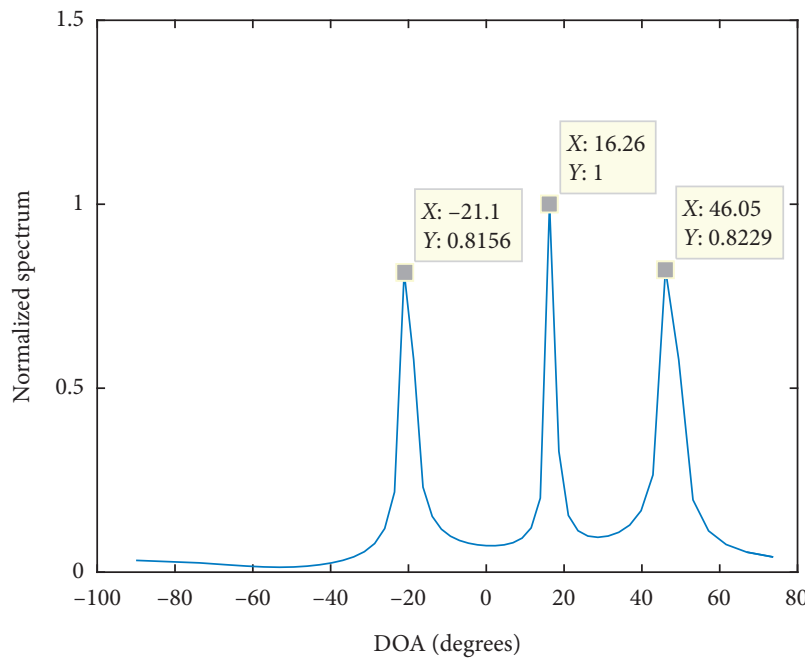

(a)

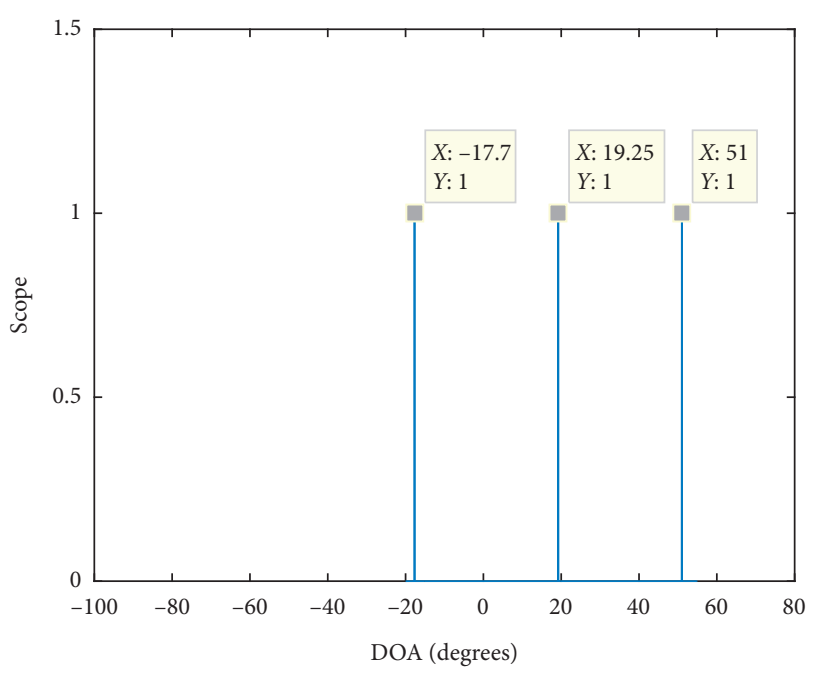

(b)

Figure 4: The result of the proposed method and classic method: (a) the estimation of real solution and (b) the result of the proposed method.

obvious that the proposed method has a better result than other classic algorithms and it should be emphasized that the proposed method can recover 10 sources which are much closer to theoretical optimal value in Theorem 1.

For off-grid case, we give a reasonable estimation of the real solution by FFT [26] and then divide the responding zone to match the real solution by a more nuanced division. In Figure 4 , the real DOA solution $\Theta^{*}=\left\{-17.7^{\circ}, 19.25^{\circ}, 51^{\circ}\right\}$, the left one shows us a rough estimation of real solution by FFT, then we divide 200 grid points around $-21.1^{\circ}, 16.26^{\circ}$ and $46.05^{\circ}$ with a $0.1^{\circ}$ interval, the right one shows the result of a more precise segmentation by the estimation.

\section{Conclusion}

In this paper, we consider the alternative function $g_{p}(x)$ to replace 0 -norm. Furthermore, the equivalence relationship between these two models is presented. Although $g_{p}(x)$ is not a smooth function, we give an analysis expression of its local optimal solution and a fixed point algorithm. Finally, we use this new alternative function to solve DOA estimation problem. Compared to some classic algorithms, the result of our method is better than the classic algorithms. In conclusion, the authors hope that in publishing this paper, a brick will be thrown out and be replaced with a gem.

\section{Data Availability}

The data used to support the findings of this study are available from the corresponding author upon request.

\section{Conflicts of Interest}

The authors declare that there are no conflicts of interest regarding the publication of this paper.

\section{Acknowledgments}

This paper was funded in part by the China Postdoctoral Science Foundation, grant numbers 2017M613076 and 2016M602775; in part by the National Natural Science Foundation of China, grant numbers 61801347, 61801344, 61522114, 61471284, 61571349, 61631019, 61871459, 61801390, and 11871392; by the Fundamental Research Funds for the Central Universities, grant numbers XJS17070, NSIY031403, and 3102017jg02014; in part by the Aeronautical Science Foundation of China, grant number 20181081003; and by the Science, Technology, and Innovation Commission of Shenzhen Municipality, grant number JCYJ20170306154716846.

\section{References}

[1] L. Wan, G. Han, L. Shu, S. Chan, and T. Zhu, “The application of DOA estimation approach in patient tracking systems with high patient density," IEEE Transactions on Industrial Informatics, vol. 12, no. 6, pp. 2353-2364, 2016.

[2] X. Wang, L. Wan, M. Huang, C. Shen, Z. Han, and T. Zhu, "Low-complexity channel estimation for circular and noncircular signals in virtual MIMO vehicle communication systems," IEEE IEEE Transactions on Vehicular Technology, vol. 69, no. 4, pp. 3916-3928, 2020.

[3] H. Wang, L. Wan, M. Dong, K. Ota, and X. Wang, "Assistant vehicle localization based on three collaborative base stations via SBL-based robust DOA estimation," IEEE Internet of Things Journal, vol. 6, no. 3, pp. 5766-5777, 2019.

[4] X. Wang, L. Wan, M. Huang, C. Shen, and K. Zhang, "Polarization channel estimation for circular and non-circular signals in massive MIMO systems," IEEE Journal of Selected Topics in Signal Processing, vol. 13, no. 5, pp. 1001-1016, 2019.

[5] F. Wen and J. Shi, "Fast direction finding for bistatic EMVSMIMO radar without pairing," Signal Processing, vol. 173, Article ID 107512, 2020.

[6] H. Chen, Y. Liu, Q. Wang, W. Liu, and G. Wang, "Two-dimensional angular parameter estimation for noncircular 
incoherently distributed sources based on an L-shaped array," IEEE Sensors Journal, 2020.

[7] Z. Zheng, Y. Huang, W.-Q. Wang, and H. C. So, "Directionof-arrival estimation of coherent signals via coprime array interpolation," IEEE Signal Processing Letters, vol. 27, pp. 585-589, 2020.

[8] H. Chen, W. Wang, and W. Liu, "Augmented quaternion ESPRIT-type DOA estimation with a crossed-dipole array," IEEE Communications Letters, vol. 24, no. 3, pp. 548-552, 2020.

[9] Y.-Y. Dong, C.-X. Dong, W. Liu, M.-M. Liu, and Z.-Z. Tang, "Scaling transform based information geometry method for DOA estimation," IEEE Transactions on Aerospace and Electronic Systems, vol. 55, no. 6, pp. 3640-3650, 2019.

[10] J. Capon, "High-resolution frequency-wavenumber spectrum analysis," Proceedings of the IEEE, vol. 57, no. 8, pp. 1408-1418, 1969.

[11] J. Li, D. Li, D. Jiang, and X. Zhang, "Extended-aperture unitary root MUSIC-based DOA estimation for coprime array," IEEE Communications Letters, vol. 22, no. 4, pp. 752-755, 2018.

[12] P. Stoica and P. Babu, "SPICE and LIKES: two hyperparameter-free methods for sparse-parameter estimation," Signal Processing, vol. 92, no. 7, pp. 1580-1590, 2012.

[13] Y. Wei and X. Guo, "Pair-matching method by signal covariance matrices for 2D-DOA estimation," IEEE Antennas and Wireless Propagation Letters, vol. 13, pp. 1199-1202, 2014.

[14] C. Zeng, S. Zhu, S. Li, Q. Liao, and L. Wang, "Sparse frame DOA estimations via a rank-one correlation model for low SNR and limited snapshots," Applied and Computational Harmonic Analysis, vol. 41, no. 2, pp. 362-383, 2016.

[15] A. Chinatto, E. Soubies, C. Junqueira et al., "L0-optimization for channel and DOA sparse estimation," in Proceedings of the International Workshop on Computational Advances in MultiSensor Adaptive Processing (CAMSAP)., IEEE, Cancun, Mexico, December 2016.

[16] J. Cai, D. Bao, P. Li, J. Cai, D. Bao, and P. Li, "DOA estimation via sparse recovering from the smoothed covariance vector," Journal of Systems Engineering and Electronics, vol. 27, no. 3, pp. 555-561, 2016.

[17] X. Li, Z. X. Wei, and Z. Ye, "DOA estimation based on sparse signal recovery utilizing weighted $l_{1}$-norm penalty," IEEE Signal Processing Letters, vol. 19, no. 3, pp. 155-158, 2012.

[18] M. Emadi, J. E. Miandji, and J. Unger, "OMP-based DOA estimation performance analysis," Digital Signal Processing, vol. 79, pp. 57-65, 2018.

[19] X. Zhang, Y. Li, Y. Yuan, T. Jiang, and Y. Yuan, "Lowcomplexity DOA estimation via OMP and majorizationminimization," in Proceedings of the 2018 IEEE Asia-Pacific Conference on Antennas and Propagation (APCAP), August 2018.

[20] Y. Chen, W. Wang, Z. Wang, and B. Xia, "A source counting method using acoustic vector sensor based on sparse modeling of DOA histogram," IEEE Signal Processing Letters, vol. 26, no. 1, pp. 69-73, 2019.

[21] F. Liu, L. Peng, M. Wei, P. Chen, and S. Guo, "An improved L1-SVD algorithm based on noise Subspace for DOA estimation," Progress In Electromagnetics Research C, vol. 29, pp. 109-122, 2012.

[22] R. Wei, Q. Wang, and Z. Zhao, "Two-dimensional DOA estimation based on separable observation model utilizing weighted L1-norm penalty and bayesian compressive sensing strategy," in Proceedings of the 2017 4th International
Conference on Information Science and Control Engineering (ICISCE), IEEE, Changsha, China, July 2017.

[23] J. Peng, S. Yue, and H. Li, "NP/CMP equivalence: a phenomenon hidden among Sparsity models $l_{0}$ minimization and $l_{p}$ minimization for information processing," IEEE Transactions on Information Theory, vol. 61, no. 7, pp. 4028-4033, 2015.

[24] S. Fourcart and H. Rauhut, A Mathematical Introduction to Compressive Sensing, Springer-Verlag, Berlin, Germany, 2013.

[25] T. T. Cai and A. Zhang, "Sharp RIP bound for sparse signal and low-rank matrix recovery," Applied and Computational Harmonic Analysis, vol. 35, no. 1, pp. 74-93, 2013.

[26] C. J. Lam and A. C. Singer, "Bayesian beamforming for DOA uncertainty: theory and implementation," IEEE Transactions on Signal Processing, vol. 54, no. 11, pp. 4435-4445, 2006. 